\title{
SUBSTRATE EFFECTS ON THE SURFACE TOPOGRAPHY OF EVAPORATED GOLD FILMS - A SCANNING TUNNELLING MICROSCOPY INVESTIGATION
}

\author{
J. VANCEA, G. REISS, F. SCHNEIDER, K. BAUER and H. HOFFMANN \\ Institut für Angewandte Physik, Universität Regensburg, Universitätssirasse 31, D.8400 Regensburg, \\ Fed. Rep. of Germany
}

Received 23 November 1988; accepted for publication 16 March 1989

Direct observation of surface roughness on metal films is a longstanding problem in thin film characterization. In this work the high quality of scanning tunnelling microscopy (STM) was used for investigation of evaporated gold films. A scanning tunnelling microscope able to scan areas up to $0.8 \times 0.8 \mu \mathrm{m}$ with high reproducibility is presented. The topography of $80 \mathrm{~nm}$ thick gold films grown under identical evaporation conditions was investigated as a function of the selected substrate material (Corning glass, silicon, $\mathrm{NaCl}$, mica and highly oriented pyrolitic graphite (HOPG)). The incipient growth mechanism on the substrate is the primary reason for the surface roughness. The real space images of the surface topography correlate very well with knowledge achieved from former growth experiments given in the literature. Moreover, very flat gold surfaces on HOPG allowed the observation of atomic corrugations in air environments.

\section{Introduction}

The surface roughness has an important influence on the physical properties of metal films as soon as the film thickness and surface roughness are of the same order of magnitude. Nevertheless, the evaluation of the surface roughness is up to now unsatisfactorily solved. For roughnesses larger than 8 $\mathrm{nm}$, transmission electron microscopy (TEM) images of surface replicas were commonly employed [1-3]. Below this range only indirect methods were available in the past.

The influence of the surface roughness on the thickness dependent resistivity was introduced in 1970 by Namba [2]. Computer fitting of the model to the experimental curve gives the surface roughness as one of the fitting parameters. This method was extensively used in our former works [4-6] for a large number of polycrystalline metal films ( $\mathrm{Au}, \mathrm{Cu}, \mathrm{Ag}, \mathrm{Ni}, \mathrm{Al}, \mathrm{Pt}$ ). The surface roughness determined on this way ranged between $0.5 \mathrm{~nm}$ for $\mathrm{Pt}$ to $10-12 \mathrm{~nm}$ for $\mathrm{Au}$ and $\mathrm{Ag}$ and was strongly correlated with the growth mechanism of the respective metal on the glass substrate. The surface roughness therefore should 
be strongly correlated with the island size prior to the coalescence to a compact film. This in turn strongly depends on substrate material and evaporation parameters.

Some disadvantages of this method should be noted here:

- A direct check of the obtained values was possible only for surface roughnesses larger than $6 \mathrm{~nm}$ with moderate accuracy $(2 \mathrm{~nm})$ by means of surface replicas method [1].

- The values obtained represent a kind of overall mean value of the surface roughness. In reality, however, detailed information about local distribution, height and lateral extension of the surface irregularities is desired in thin film investigations.

The discovery of the STM [7] and the explosive growth of applications in the forthcoming years give a change to remove this handicap. Real space images of the surface topography in vacuum, air and liquid environments were obtained for thin films of various metals. Very attractive for thin film routine work is STM in air.

Marchon and coworkers [8] applied the STM for characterization of $100 \mathrm{~nm}$ thick gold film surfaces deposited on mica. Jaklevic and coworkers [9] used the images of very thin gold coatings for the characterization of the underlying insulating surfaces. Some examples of surface topography for gold films evaporated on silicon are given by Laiho and coworkers [10]. The most spectacular result was the observation (in air) of the atomic corrugation on the surface of Au(111) $250 \mathrm{~nm}$ thick gold epitaxial films on cleaved mica substrates by Hallmark and coworkers [11].

Most of the investigations in air have been made for rather inert materials (i.e. HOPG and gold), since the influence of the adsorbates on the STM images is yet not well enough understood [12].

Despite of the so far encouraging results, the problem of the surface topography of evaporated gold films is rather unsatisfactorily treated up to now. The few examples given in the literature cannot be compared with each other because of different or in some cases unspecified deposition parameters.

The recent work of Chidsey and coworkers [16], however, demonstrated the relevance of systematic thin film investigations using STM. These authors studied the influence of the substrate temperature on the epitaxial growth of gold films on mica. Relevant STM images related to overviews of large areas but also atomic scale topography (atomic steps, dislocations) were performed, correlating well with the simultaneous investigations by $\mathrm{X}$-ray diffraction and TEM.

In this work we discuss comparative STM investigations of gold film surfaces in air. The deposition parameters are identical for all investigated films. The films, however, were deposited onto various substrates. This way, we easily obtained a synoptic view of the substrate induced surface topography for gold coatings. 


\section{Experimental technique}

\subsection{Apparatus}

In order to obtain reproducible images a STM with a suitable scanning area, high resolution but first of all of very high stability was necessary. The design of our scanning tunnelling microscope is sketched in fig. 1.

Two Invar manufactured differential screws ( 2 and 4$)$ and the piezo-scanner unit 5 are mounted on the massive stainless steel block 1 of the apparatus. Sample 6 is mounted onto the differential screw 4 which provides the coarse mechanical approach towards the electrochemically etched tungsten tip mounted on the single tube scanner [13]. The fine mechanical approach consists of the differential screw 2 ( $50 \mu \mathrm{m}$ per turn) pressing onto arm 3 by means of an elastic metallic membrane of adequate thickness. This way, the gap machined into the stainless steel block 1 can be mechanically controlled with very high accuracy (better than $50 \AA$ ). This approach is very reliable, easy to operate and gives rise to a high mechanical stability of the tunnelling gap.

The apparatus was mounted on top of a stack of stainless steel plates separated by Viton spacers (pocket size design [14]) and altogether located on top of a stack of two stone plates separated by rubber rings; this system supresses very well vibrations with frequencies above $2 \mathrm{~Hz}$. The electronics, realized in the known conventional manner, is computer controlled. A per-

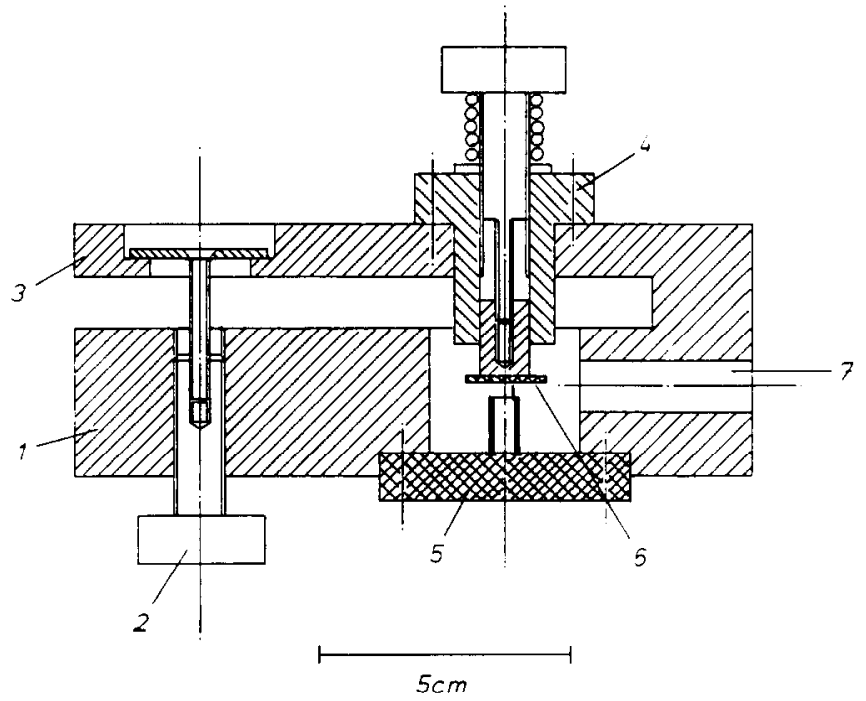

Fig. 1. Schematic view of our STM. (1) Stainless steel block, (2) differential screw (fine approach), (3) elastic stainless steel arm, (4) differential screw (coarse approach), (5) scanning unit with single tube scanner and tip, (6) sample, (7) view for coarse approach control. 


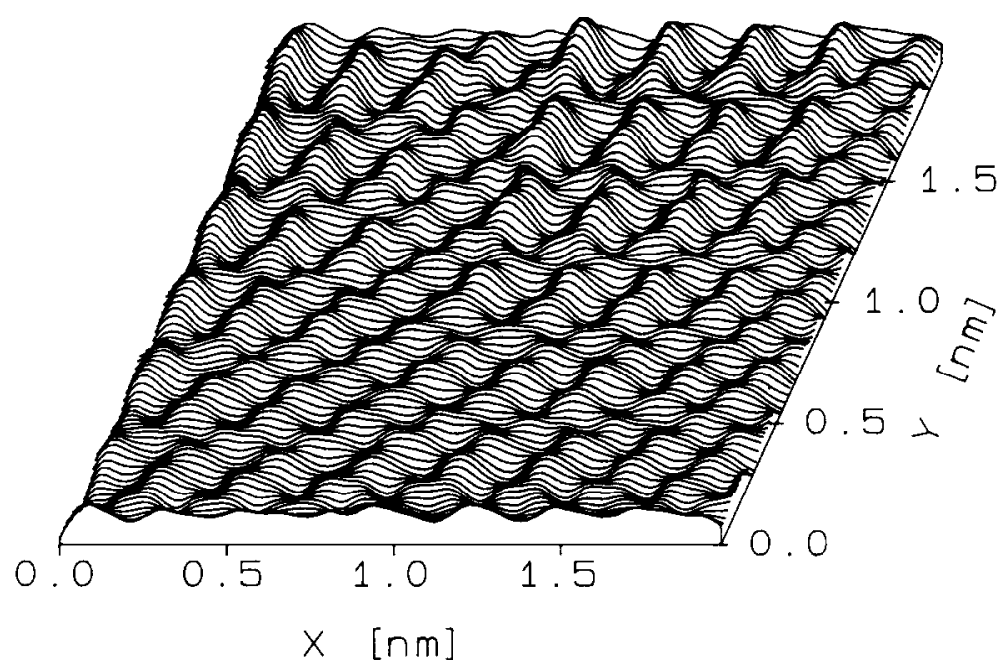

Fig. 2. STM constant height picture of a $20 \times 20 \AA$ area on HOPG. The well resolved hexagonal corrugation of the $\beta$-sites can be observed.

sonal computer provides for the variation of the $X, Y$ scanning voltages and data acquisition. The maximal scanning rate was $100 \mathrm{~nm} / \mathrm{s}$. Typical acquisition times vary from about $10 \mathrm{~s}$ for a $20 \times 20 \AA$ constant height mode picture to about $25 \mathrm{~min}$ for a $0.8 \times 0.8 \mu \mathrm{m}$ constant current mode picture. The distances in $X$ and $Y$ were determined from the applied piezo-voltages calibrated against graphite (HOPG, Union Carbide). A typical constant height picture (line plot) of HOPG is given in fig. 2.

The hexagonal atomic corrugation of the graphite with the $2.46 \AA$ interatomic spacing is very well resolved by our microscope. The resolution of the tungsten tips corresponds consequently to the accepted standard level of STM.

The calibration factor for the elongation in the $Z$-direction of the single tube fabricated from P1-60 $\left(d_{31}=175 \times 10^{-12} \mathrm{~nm} / \mathrm{V}\right.$, see ref. [15] $)$ was determined by interferometry. For this purpose a gold film on $\mathrm{Si}(100)$ mounted on a piezo-tube (in the following called piezo-holder) was adjusted in a Tolansky microscope so that multiple interference occurs. An applied piezovoltage gives rise to a variation of the distance between film and the interferometric device and therefore to a corresponding shift of the interference lines. This allows the calculation of the elongation factor $(\mathrm{nm} / \mathrm{V})$ of the piezo-holder. The same arrangement (gold film with piezo-holder) was thereafter mounted onto the sample unit of our STM. The microscope was adjusted to a common tunnelling distance $\left(10^{7} \Omega\right)$ used for the operation in the constant current mode. Then the sample was moved towards the tunnelling tip by means of applied voltages on the piezo-holder. The feedback control of the STM, however, maintained the tunnelling distance constant with appropriate volt- 
ages applied to the $Z$-electrodes of the tip single tube scanner. With these voltages and the elongation factor of the piezo-holder, the value for the calibration of the Z-scale in the STM images can be accurately determined. This method therefore permits the calibration of the piezo-scanner in situ, i.e. mounted in the scanning unit with attached tunnelling tip.

A very useful feature of the microscope is a very simple operation in a zoom modus. The operator can select immediately a new scanning frame onto the foregoing (larger) scan area and start a new scan with much higher resolution. The reproducibility of the topographical imaging should be sufficiently good for the operation in this modus; for our microscope the thermal drift was commonly smaller than $0.6 \mathrm{~nm} / \mathrm{min}$, provided the temperature of the sur-
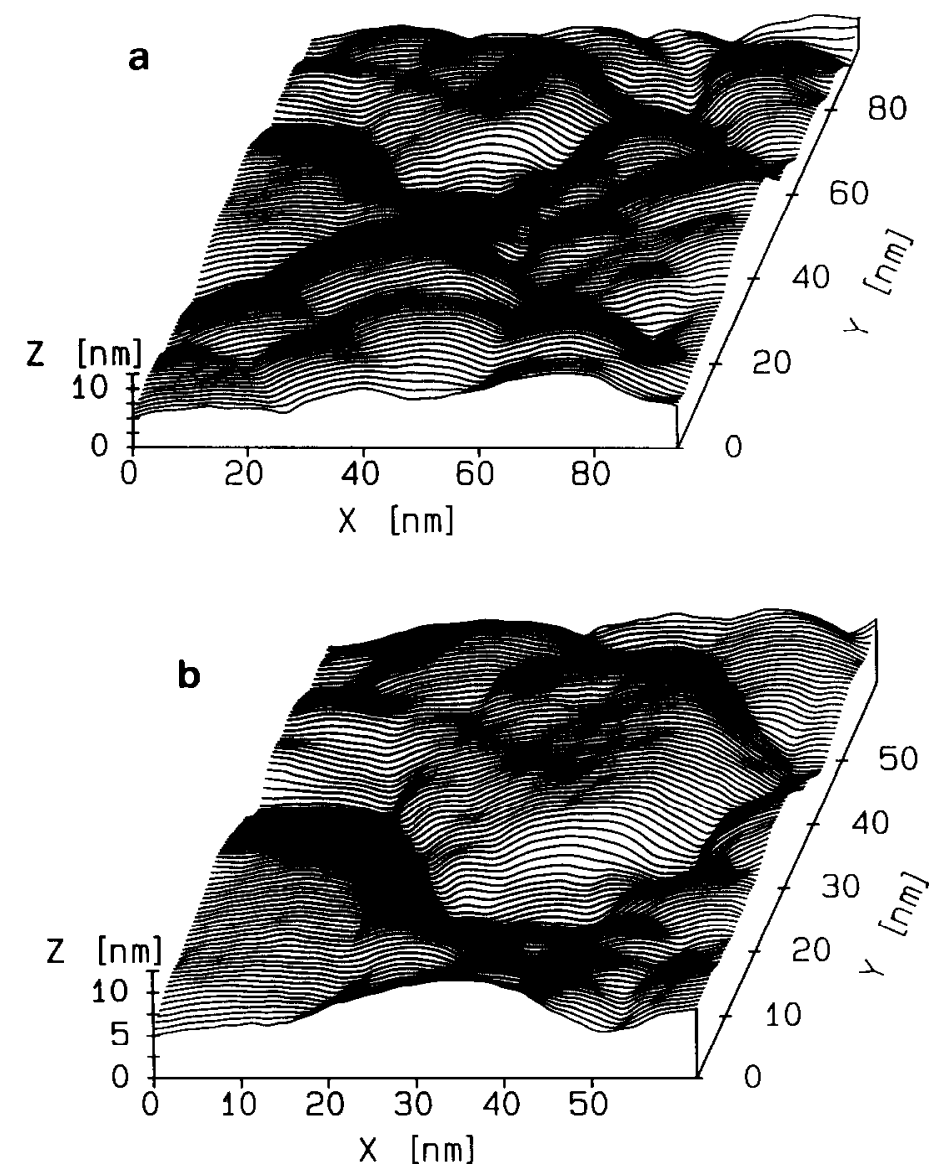

Fig. 3. Zoom feasibility of our STM exemplified with the surface topography of a Au(12 $\mathrm{nm})-\mathrm{Nb}(12.5 \mathrm{~nm})-\mathrm{Au}(6 \mathrm{~nm})$ sandwich film on $\mathrm{Si}(100)$ : (a) $90 \times 90 \mathrm{~nm}$ area scan; (b) a new zoom-scan of the hill located at the upper-left corner of (a). 
roundings changed not faster than $2^{\circ} \mathrm{C} / \mathrm{h}$. The zoom feasibility of our STM is demonstrated in fig. 3.

The surface topography shown in fig. $3 \mathrm{a}$ was observed on the $90 \times 90 \mathrm{~nm}$ upper area of a sandwich film consisting of a $12 \mathrm{~nm}$ thick gold film on etched (100) silicon substrate superimposed with a $12.5 \mathrm{~nm}$ thick $\mathrm{Nb}$ film and finally again covered with a $6 \mathrm{~nm}$ thick gold film. The evaporation parameters were: $10^{-8} \mathrm{mbar}, 0.1 \mathrm{~nm} / \mathrm{s}$ and $300 \mathrm{~K}$ substrate temperature. The surface topography has a particular shape with a few hills of about $8 \mathrm{~nm}$ in height and $30 \mathrm{~nm}$ lateral extent. The picture in fig. $3 \mathrm{~b}$ is a zoom-scan of the hill located at the upper-left corner of the fig. 3a. On the time scale there are about $20 \mathrm{~min}$ between the two pictures. Nevertheless, each detail of fig. $3 a$ can be found on the magnified survey in fig. $3 \mathrm{~b}$. Thus effects concerning piezo-creep or thermal drifts seem to be not significant for large scale topographical imaging.

The shape (especially the sharpness) of the tunnelling tip is the main factor limiting the resolution of large topographical features. In fig. 3b, structures with a width and depth of about $5 \mathrm{~nm}$ can be clearly recognized. As demonstrated later (fig. 7a) cleavage steps at large vertical and lateral extension can be well resolved. In our experiment, only tips with a radius smaller than $100 \mathrm{~nm}$ and cone angles below $40^{\circ}$ have been used.

In order to obtain reproducible (reliable) images usually some "dummy" scans have to be performed. During these scans, both surface as well as tip contaminations are removed. This was clearly demonstrated by Besocke et al. [12] for Si surfaces. Especially for gold surfaces, only a contamination in the monolayer range is to be expected. Therefore, the large topographical features discussed in this paper are not essentially influenced by contamination.

Additionally, large local variations of the tunnelling barrier $\Phi$ are not expected. Even in the presence of varying barrier heights (by a factor two or three for example), the tip-surface distance would change only by $1-2 \AA$.

Finally some remarks concerning the adjusted sample-tip distances during the operation of the microscope should be given. This distance, mostly given in terms of tunnelling resistance, influences very strongly the lateral and vertical resolution of the apparatus.

During the operation of our microscope we used the following values for the tunnelling resistance: $10^{7} \Omega$ (typically $100 \mathrm{mV}, 5 \mathrm{nA}$ ) for surface topography images of gold, $10^{6} \Omega$ (typically $20 \mathrm{mV}, 5 \mathrm{nA}$ ) for atomic corrugation images on graphite and $10^{5} \Omega$ (typically $2 \mathrm{mV}, 10 \mathrm{nA}$ ) for atomic corrugation images on Au.

\subsection{The preparation of samples}

Five substrate materials were selected: Corning glass, Si(100) wafers, mica, $\mathrm{NaCl}$ and HOPG. 
The Corning glass substrates were rinsed in an ultrasonic bath with aceton, water and ethanol and then cleaned with the common Balzers cleaning set. The optically polished $\mathrm{Si}(100)$ wafers were etched in $\mathrm{HF}$ and then rinsed with water and ethanol. The $\mathrm{NaCl}$ single crystals were simply cleaved in air by a sharp blow with a knife edge just before their insertion in the evaporation system. Mica and HOPG were freshly cleaved in air and immediately mounted into the evaporation system.

In order to obtain comparable results, all substrates were treated in the same manner: They were mounted together on the same holder, about $30 \mathrm{~cm}$ above the evaporation source (for details see ref. [4]) and baked out at $500 \mathrm{~K}$ in UHV $\left(10^{-9} \mathrm{mbar}\right)$ for some days. Subsequently these were simultaneously coated with an $80 \mathrm{~nm}$ gold film at the same substrate temperature $(500 \mathrm{~K})$ and at an evaporation rate of $0.1 \mathrm{~nm} / \mathrm{s}$. Due to the low heating power (100 W) necessary for the selected evaporation rate, the deposition pressure could be maintained at $10^{-8}$ mbar.

The films then were annealed at $500 \mathrm{~K}$ for $4 \mathrm{~h}$ and subsequently stored at $300 \mathrm{~K}$ in the ultrahigh vacuum chamber up to the STM investigations. Thus the temperature during the post-deposition treatment did neither exceed the deposition temperature nor the commonly accepted value for recrystallization of polycrystalline gold films $(600 \mathrm{~K})$ [33]. Consequently, only the common annealing of point-defects should occur during this treatment, whereas the large scale topographical features can be expected to remain unchanged. The different surface features imaged by STM therefore should be mainly due to the growth mechanisms of these films.

We obtained therefore gold film of identical thickness and evaporation parameters. The comparative study of the surface topography of these films on the selected substrates will be discussed in the following.

\section{Results and discussion}

The observed surface topographies are classified into three categories:

(a) Rough surfaces, i.e. the film surface map presents an overall hill landscape, much stronger than the roughness of the substrate.

(b) Smooth surfaces, i.e. the surface of the thin film is much smoother than the substrate. The surface map mainly shows features of the underlying substrate together with much smaller features of own topography. These two contributions can be clearly distinguished from each from other.

(c) Mixed surfaces, i.e. both smooth and rough distinct regions can be observed on the film surface. This is mainly typical for inhomogeneous surfaces caused by different local growth processes on the substrate. 
(a) Rough surfaces

\section{Au on glass}

Fig. 4 shows a typical $0.3 \times 0.3 \mu \mathrm{m}$ scan of a gold film on fire polished Corning glass substrate (Corning 7039). The surface of the gold films on glass is very rough (mountain-like landscape). Hills of various heights and lateral extension are statistically distributed on the surface. A typical $Z$ corrugation(roughness) of $11 \mathrm{~nm}$ can be estimated, but also hills of about 6 or $17 \mathrm{~nm}$ can be observed. It should be noted that the present authors evaluated mean roughness values of $11 \mathrm{~nm}$ from the thickness dependent resistivity of gold films on Corning glass [5]. The lateral extension of the surface irregularities varies roughly between 20 and $40 \mathrm{~nm}$. Moreover, fig. 4 shows that the surface irregularities of gold films on glass are rather uncorrelated.

The present authors $[4,5]$ concluded from resistivity measurements that the size of islands prior to the coalescence to a compact film is the main reason for the surface roughness (this corresponds roughly with the onset thickness of the electrical continuity). The landscape in fig. 4 offers a similar impression. The liquid-like coalescence of gold on glass is a well-known phenomenon. A wide-ranging statistical distribution of the surface irregularities, i.e. a weak correlation between surface structures, is therefore a logical consequence. The nucleation of gold on glass was extensively studied [17], last by Anderson and coworkers $[18,19]$. They reported a large spread in the island sizes accompanied by a liquid-like coalescence at critical thicknesses $(8-12 \mathrm{~nm})$ comparable with those of our resistivity measurements.

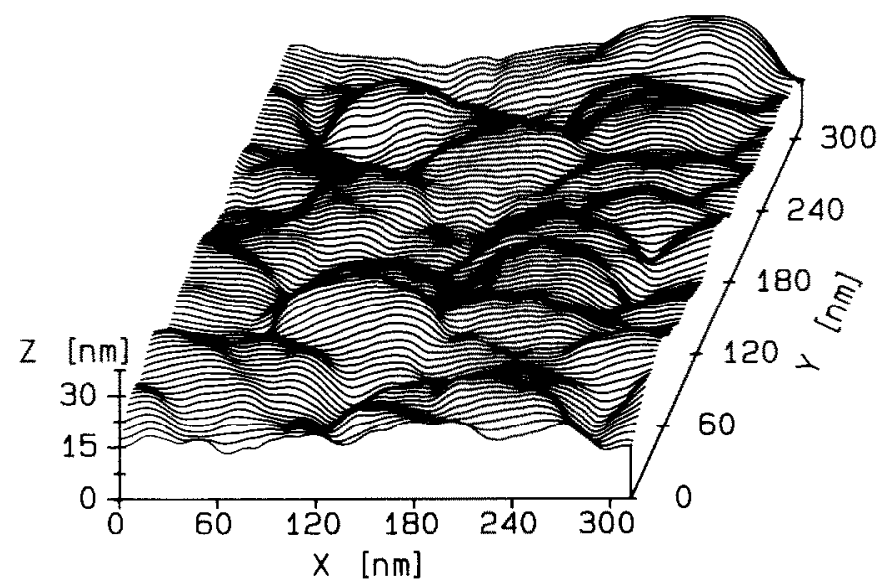

Fig. 4. STM image of a $A u(80 \mathrm{~nm})$ film surface on Corning glass. These films show uncorrelated rough surfaces. 
Au on precoated glass

In the following, we give for the first time direct evidence that island sizes just prior to coalescence represent the main reason for the roughness of polycrystalline metal films. For this purpose, chromium nucleation centers were evaporated onto the glass substrates just before the deposition of $\mathrm{Au}$ in order to inhibit the island growth. Chromium is an oxygen active material leading to very stable nucleation centers on glass or oxidized silicon.

Numerous experiments in our laboratory at low chromium coverages (thicknesses smaller than $1.5 \mathrm{~nm}$ ) did not show any influence on the crystallite size of the compact gold films apart from a more homogeneous distribution of crystallite sizes. Note that the crystallite size usually cannot be identified with the island size.

On the contrary, the onset thickness of electrical continuity (critical thickness where coalescence occurs) was drastically reduced to values of 2 to $4 \mathrm{~nm}$, dependent on the density of chromium nucleation centers on the glass substrate. A strong reduction of the island sizes can be assumed and thereupon a reduction of the surface roughness to values below $4 \mathrm{~nm}$, suggesting that the island coagulate to larger flat crystallites.

The surface topography of a gold film on Corning glass previously covered with $1.5 \mathrm{~nm}$ chromium is illustrated in fig. 5. This picture confirms our concept regarding the surface roughness of polycrystalline metal films. The hill landscape, presented in fig. 4 , is drastically levelled out due to the existence of the chromium nucleation centers. Large areas with surface roughnesses between 2 and $3 \mathrm{~nm}$ can be observed in fig. 5. Only a few level differences of 6 $\mathrm{nm}$ scattered over these smooth areas can be recognized.

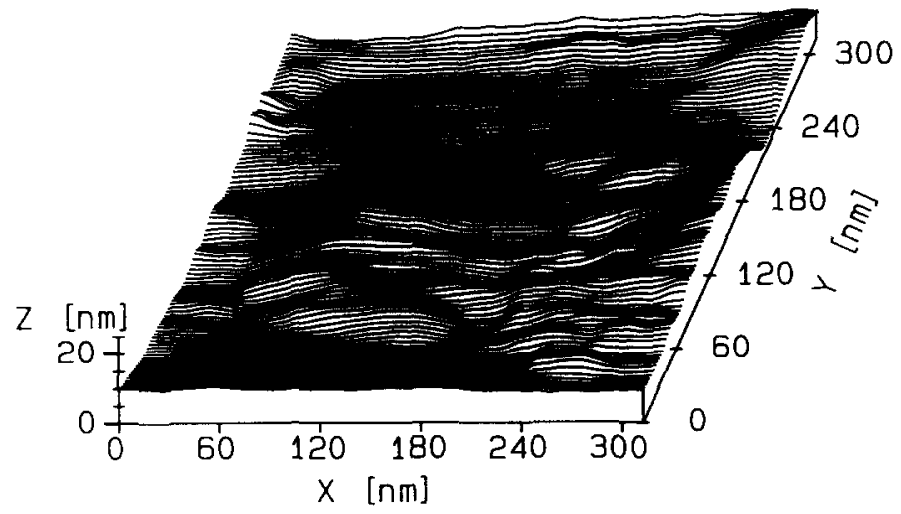

Fig. 5. Surface topography of a $\mathrm{Au}(80 \mathrm{~nm})$ film on precoated Corning glass substrate. The presence of the $\mathrm{Cr}$ nucleation centers leads to the smoothing of the gold films on glass. 


\section{Au on Si(100)}

Gold films are known to coagulate during the growth on silicon substrates. The picture of the surface topography of such coatings is a popular example given by many authors of STM papers $[10,20-22]$ or by manufacturers in order to demonstrate the quality of the apparatus. Unfortunately, the results are not comparable with our experiment due to the reasons given in the introduction of this paper.

The surface diffusion of $\mathrm{Au}$ on $\mathrm{Si}$ is much larger than on glass and therefore rather an enhancement in the island size with the consequence of an enhanced surface roughness can be expected. The majority of STM-scans given in the literature for $\mathrm{Au}$ on $\mathrm{Si}$ relate to film thicknesses below $10 \mathrm{~nm}[10,21,22]$. All these films have a cluster-like structure with cluster sizes of about 10 to $30 \mathrm{~nm}$ lateral extension and 3 to $10 \mathrm{~nm}$ in height even at these low film thicknesses.

In fig. 6 we show the surface topography of our gold films, deposited on $\mathrm{Si}(100)$. As expected we observe a hill-like landscape, with an average height variation of about $16 \mathrm{~nm}$, i.e. the surface roughness is enhanced compared to Au films on glass substrates. Moreover, the lateral extension of the hillocks is increased from $40 \mathrm{~nm}$ for gold on glass to $80 \mathrm{~nm}$ for gold on $\mathrm{Si}(100)$. The existence of an enhanced surface roughness even for films on very homogeneous and flat substrates as in the case of $\mathrm{Si}(100)$ is clearly due to the enhanced island growth on this material.

In contrast to the films on glass, the surface roughness of $\mathrm{Au}$ on $\mathrm{Si}(100)$ shows a high degree of correlation: The dimensions of the hillocks are more homogeneously distributed than for glass substrates, but at the same time much larger than observed for clustered (islandized) films. A possible explana-

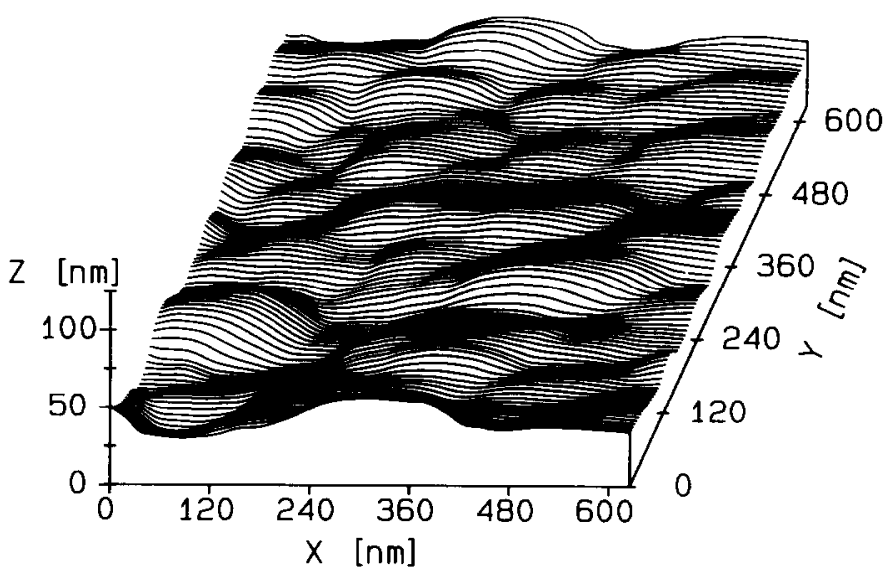

Fig. 6. Typical surface topography of $\mathrm{Au}(80 \mathrm{~nm})$ film surfaces on $\mathrm{Si}(100)$. The films behave a correlated rough surface. 
tion may be supported by the recently reported growth mechanism of $\mathrm{Au}$ on $\mathrm{Si}(111)$ [23]: At small thicknesses a kind of silicide ( $\mathrm{Au}-\mathrm{Si}$ alloy) clusters are formed on the $\mathrm{Si}(111)$ surface. For films grown at RT and thinner than $10 \mathrm{~nm}$, such silicide particles with a diameter of about $30 \mathrm{~nm}$ have been observed [23]. With increasing film thickness new layers of pure gold crystallites are formed.

On the other hand, the existence of silicide particles on silicon gives rise on an artificial roughening of the substrate with bumps of relatively regular sizes which can be much larger for our heated substrates due to the temperature-enhanced surface diffusion. This artificially roughened substrates are responsible for the observed roughness of the $80 \mathrm{~nm}$ thick gold films on $\mathrm{Si}(100)$.

\section{(b) Smooth surfaces}

\section{$A$ on $\mathrm{NaCl}(100)$}

Surfaces of remarkable smoothness of films condensed on $\mathrm{NaCl}$ and graphite (HOPG) have been observed. The growth of noble metals on the (100) cleavage planes of alkali halides was intensively studied both by thin film and surface investigators [24-26]. A large range of epitaxial temperatures for $\mathrm{Au}$ on $\mathrm{NaCl}(100)$, varying from $500 \mathrm{~K}$ [25] up to $670 \mathrm{~K}$ [26] have been reported in the literature. The surface of epitaxial gold films on $\mathrm{NaCl}(100)$ is generally assumed as smooth in the atomic range over large areas.

On the other hand, Jacklevic et al. [9] exploited the smoothness of very thin $(2.5 \mathrm{~nm})$ gold films deposited at low substrate temperatures $(77 \mathrm{~K})$ in order to obtain STM images of the underlying $\mathrm{NaCl}$ substrate cleaved in air by a sharp blow with a knife edge. Smooth regions free of any structure with occasional cleavage steps of various heights (maximum $8 \mathrm{~nm}$ ) of the $\mathrm{NaCl}$ single crystal were observed in the $100 \times 70 \mathrm{~nm}$ large area scans. Only multiple-atom height cleavage steps could be imaged on the smooth area $(20 \times 14 \mathrm{~nm})$ scans.

We cleaved the $\mathrm{NaCl}$ single crystal substrates by the same procedure as Jaklevic and coworkers [9]. The result of a large area scan $(0.6 \times 0.6 \mu \mathrm{m})$ of the gold film surface on these substrates is given in fig. $7 \mathrm{a}$.

The film surface is smooth to a degree that allows an excellent overlook of surface features of the underlying substrate. The staircase-landscape on the right of the picture, whose steps have a relative height to the adjacent steps a multiple of about $1.5 \mathrm{~nm}$ (i.e. $1.5,15,45$, and $60 \mathrm{~nm}$ ), is clearly due to the (100) cleavage steps of $\mathrm{NaCl}$. No signs of the gold overlayer can be seen in this picture, despite of the $80 \mathrm{~nm}$ film thickness and the much higher deposition temperature.

We consequently performed a zoom-mapping on the very smooth area located around $X=240 \mathrm{~nm}$ and $Y=120 \mathrm{~nm}$ in fig. $7 \mathrm{a}$ with much higher resolution. The result is given in fig. $7 \mathrm{~b}$. The apparently very smooth area of the foregoing large area scan shows now a substructure in a very fine microscopical height scale. The relief map shows again an overall maximum 

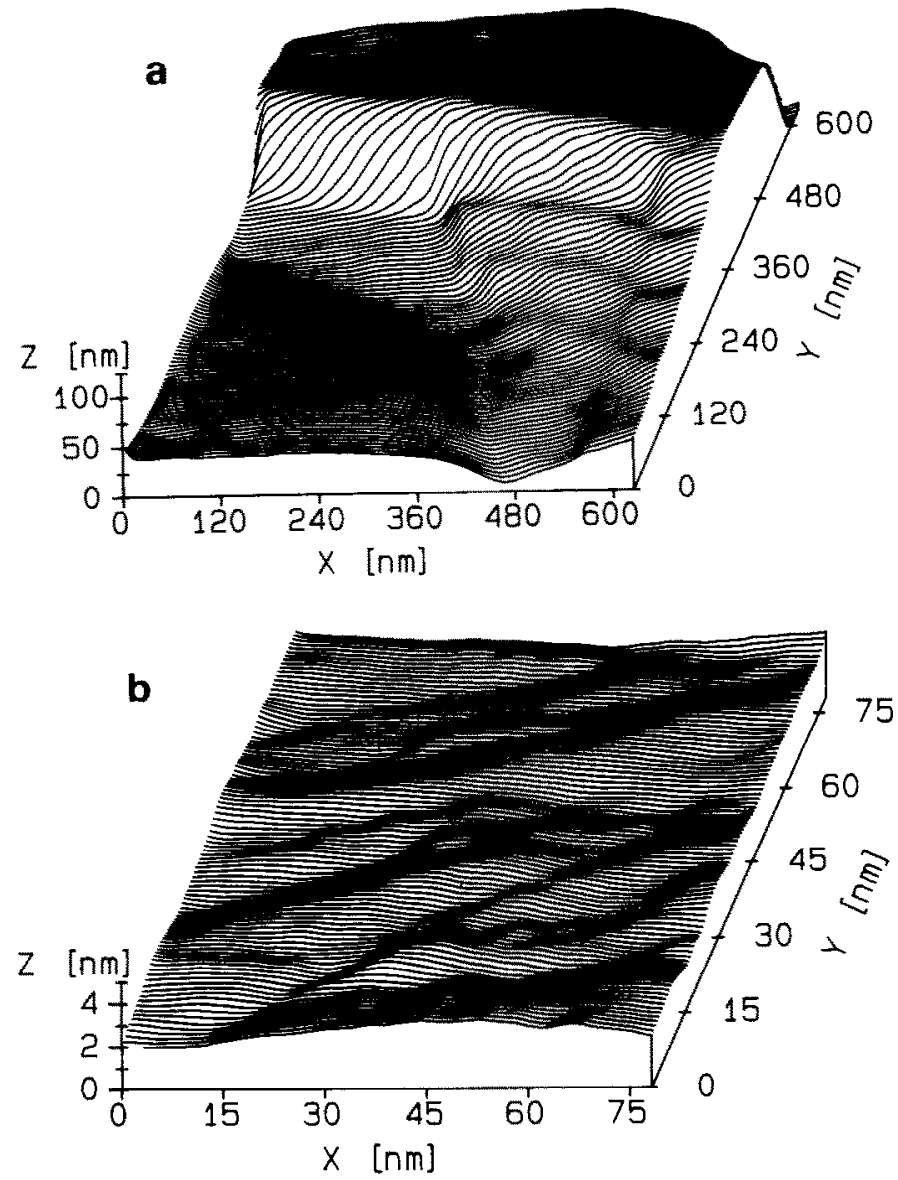

Fig. 7. Surface topography of a $\mathrm{Au}(80 \mathrm{~nm})$ film surface on cleaved (100) $\mathrm{NaCl}$ (a) Large area scan $(0.6 \times 0.6 \mu \mathrm{m})$. The $(100)$ cleavage steps of $\mathrm{NaCl}$ can be clearly observed. (b) Zoom-scan of the area located at around $X=240 \mathrm{~nm}, Y=120 \mathrm{~nm}$ of (a); with exception of the features attributed to atomic (100) cleavage steps no sign of the gold film can be recognized; the film surface is very smooth (in the $1 \AA$ range).

level difference of about $1.5-1.8 \mathrm{~nm}$. Moreover, this smooth field shows a lot of smaller (but still in the $10 \mathrm{~nm}$ range) areas, with level difference between adjacent areas of about 5-6. . Assuming atomically flat gold films, this could be related to atomic (100) cleavage steps of $\mathrm{NaCl}$ (the (100) atomic level spacing in $\mathrm{NaCl}$ is $5.65 \AA$ ), i.e. the applied knock on the $\mathrm{NaCl}$ single crystal has produced (100) cleavage steps not only in the "macroscopic" range; this naturally propagates up to the atomic scale. This interpretation, however, needs further confirmation since a propagation of such steps through a $80 \mathrm{~nm}$ thick film seems to be hardly acceptable. 
The most important result, however, is the exceptional smoothness of 80 $\mathrm{nm}$ gold films (in the $1.0 \AA$ range) deposited at $500 \mathrm{~K}$ on the (100) cleavage planes of $\mathrm{NaCl}$.

\section{Au on HOPG}

Little work concerning the growth of gold films on single crystalline graphite has been reported in the literature. This is rather suprising, since the advantage of hexagonal-packed substrates for the (111) oriented growth of the fcc metals was generally recognized. Moreover, extensive work concerning the epitaxial growth of gold on similar structures (mica, molybdenite) was done in the past [26-28].

In 1970 Darby and Wayman [29] made a first attempt to the systematic study of gold films evaporated at $0.1-0.2 \mathrm{~nm} / \mathrm{s}$ in $10^{-9} \mathrm{mbar}$ on single crystalline graphite substrates. Referring to this work, an imperfect epitaxial structure, but certainly with a very strong (111) fiber texture, can be expected for the samples on HOPG.

In connection with STM imaging of small metal clusters (in UHV) on the basal plane of graphite substrates, a quite interesting result was recently reported by Ganz et al. [30]. Very stable Au dimer clusters located on the $\beta$-sites were detected,whose bond length of $2.47 \AA$ is very close to the well-known value of $2.46 \AA$ for the separation of the $\beta$-sites on the HOPG surface. Regarding this result a stable incipient gold monolayer on the $\beta$-sites of the HOPG substrate can be tentatively conceived. This will in turn contribute to the building of a smooth overlayer surface.

A typical picture of gold overlayers discussed in this work is shown in fig. 8a. The smoothness of the gold coating is better than the topographical features of the underlying graphite substrate. The level differences located in the region located around $X=45 \mathrm{~nm}, Y=45 \mathrm{~nm}$ are related to cleavage steps of graphite. These level differences amount to about $6 \AA$ or multiples of this and therefore can be correlated with the separation value of $6.7 \AA$ of the equivalent basal planes of the graphite lattice.

In order to prove the orientation of the surface, we performed $20 \times 20 \AA$ constant height pictures of the very smooth area located at $X=30 \mathrm{~nm}, Y=60$ $\mathrm{nm}$. Additionally, the tip-sample distance was drastically reduced to tunnelling resistances of $10^{5} \Omega$.

The result is given in fig. $8 \mathrm{~b}$. On the left side, the close-packed (111) hexagonal corrugation of gold can be clearly observed. The perturbation on the right can be due to lattice defects or much simpler to a sudden drift of the apparatus (line-scanning here was performed in the $Y$-direction). The estimated atomic spacing of $2.8 \AA$ (calibrated against graphite measurements) corresponds fairly well with the known gold interatomic distance of $2.88 \AA$ and with the result in air of Hallmark et al. [11] for epitaxial gold films on mica. The corrugation amplitudes in fig. $8 \mathrm{~b}$ ranged to $2.7 \%$ of the tunnelling 

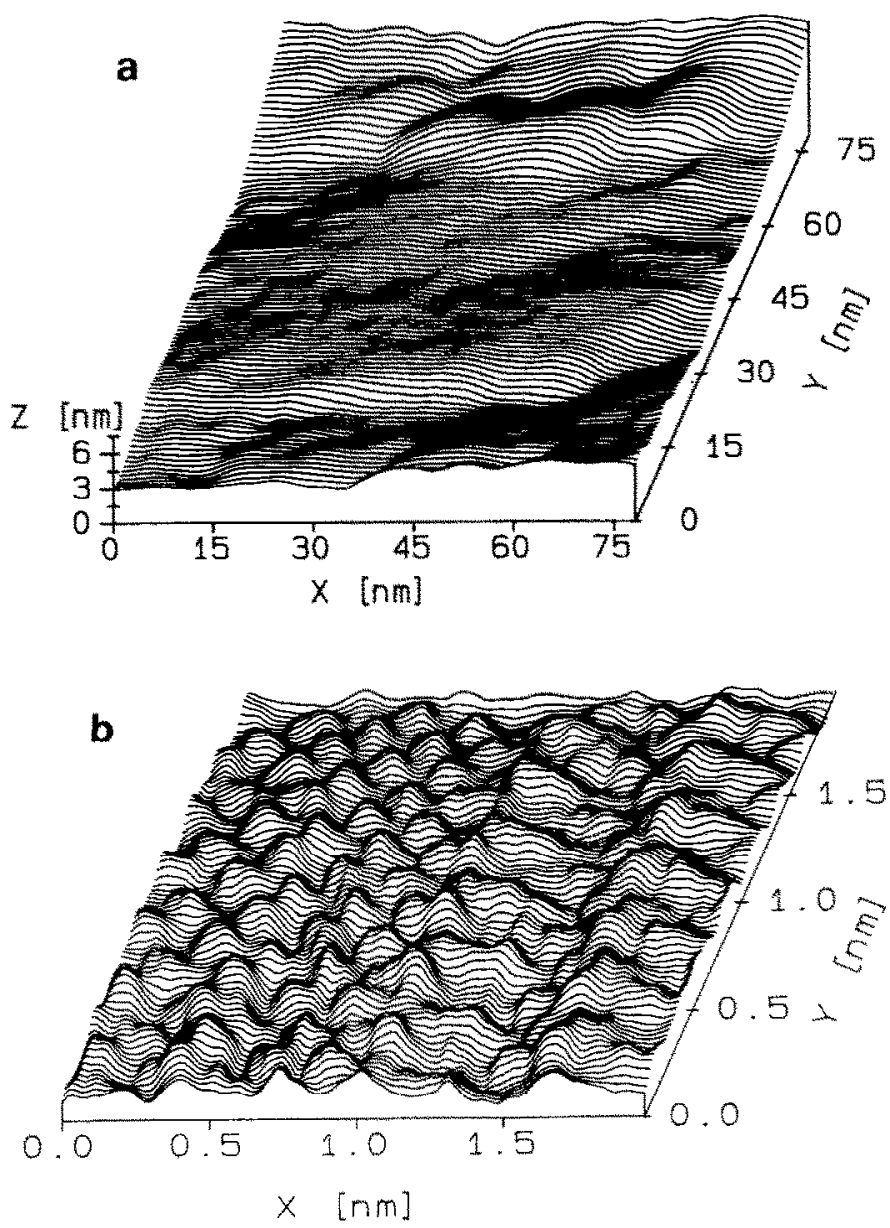

Fig. 8. Surface topography of a Au(80 nm) film surface on HOPG:(a) Large area scan $(75 \times 75$ nm). The noticeable topographical features are mainly due to the cleavage steps of basal planes in HOPG. (b) Constant height picture of the $20 \times 20 \AA$ area located at $X=30 \mathrm{~nm}, Y=60 \mathrm{~nm}$ of (a). The (111) hexagonal corngation of gold with an atomic spacing of $2.9 \AA$ can be observed on the left.

current (11 nA) and consequently is much smaller than the common value of $10 \%$ observed for constant height HOPG pictures (fig. 2).

Although Hallmark and coworkers [11] did not exclude an epitaxial adsorbed layer on an (111) gold surface, the film beneath has to be epitaxial too. An adsorbate layer on Au, however, seems to be unlikely, because these authors observed the same corrugation on the same films in UHV.

Therefore, we have shown a close-packed corrugation of gold in air on very flat, small areas of (111) textured films on HOPG. The main purpose of this 
work being not atomic resolution on metal surfaces, this attempt was not carried out for the other systems discussed here.

\section{(c) Mixed surfaces}

\section{Au on mica}

Mica is one of the favourite materials for epitaxial deposition studies and consequently some work concerning the oriented growth of noble metals on this material already exists [26-28].

According to results published in the literature $\mathrm{Au}$ and $\mathrm{Ag}$ show for example a higher tendency for orientation on $\mathrm{NaCl}$ than on mica. Reichelt and Lutz [31] observed for vacuum evaporated $\mathrm{Ag}$ on mica at substrate temperatures higher than $650 \mathrm{~K}$ nearly perfect epitaxy for $\mathrm{Ag}$ (untwinned single crystals), but only partial epitaxy for Au films. Nevertheless, highly oriented (111) textures with six-fold symmetry were observed for gold films condensed at substrate temperatures higher than $570 \mathrm{~K}$. Surface topography studies with STM for gold on mica were reported by Marchon et al. [8] and Hallmark et al. [11].

A smoothly rolling hill structure with hill sizes in the range of $10 \mathrm{~nm}$ lateral extension and a few nanometer in height were observed for films grown at room temperature [7]. After annealing in air at $520 \mathrm{~K}$, this topography changed to an atomically flat one with some scattered bumps and trenches attributed to defect structures in the mica substrate or residual grain boundaries in gold films.

Hallmark et al. [11] performed STM investigations of (111) epitaxial gold films (film thickness $=250 \mathrm{~nm}$ ) evaporated in $10^{-6}$ mbar onto cleaved mica substrates maintained at $570 \mathrm{~K}$, i.e. in accord with the prescriptions of Reichelt and Lutz [31]. After $\mathrm{Ar}^{+}$sputtering and post-annealing in UHV at $630 \mathrm{~K}$, very smooth surfaces with atomic monolayer steps of about $5 \mathrm{~nm}$ lateral extension were imaged. Finally, on these steps the hexagonal atomic corrugation of $\mathrm{Au}(111)$ was excellently resolved both in air and in UHV.

The STEM investigation of the epitaxial growth of gold on mica by Chidsey and coworkers [16] deals with 50,120 and $480 \mathrm{~nm}$ thick films grown by e-gun evaporation in $10^{-6}-10^{-7}$ mbar and $0.1 \mathrm{~nm} / \mathrm{s}$ on air-cleaved muscovite substrates; the substrate temperatures was $300,428,503$ and $573 \mathrm{~K}$ respectively. For temperatures higher than RT, they observed large flat crystallites containing holes, dislocations and twins.Good epitaxial orientation was obtained only for a substrate temperature of $503 \mathrm{~K}$. With increasing substrate temperature, the films were even flatter but the quality of epitaxy was poorer. This result appears astonishing and contradicts the previously discussed growth experiments of Reichelt and Lutz [31]. For the relevant temperature of $503 \mathrm{~K}$, high atomically flat plateaus of some hundred $\mathrm{nm}$ lateral extension separated 

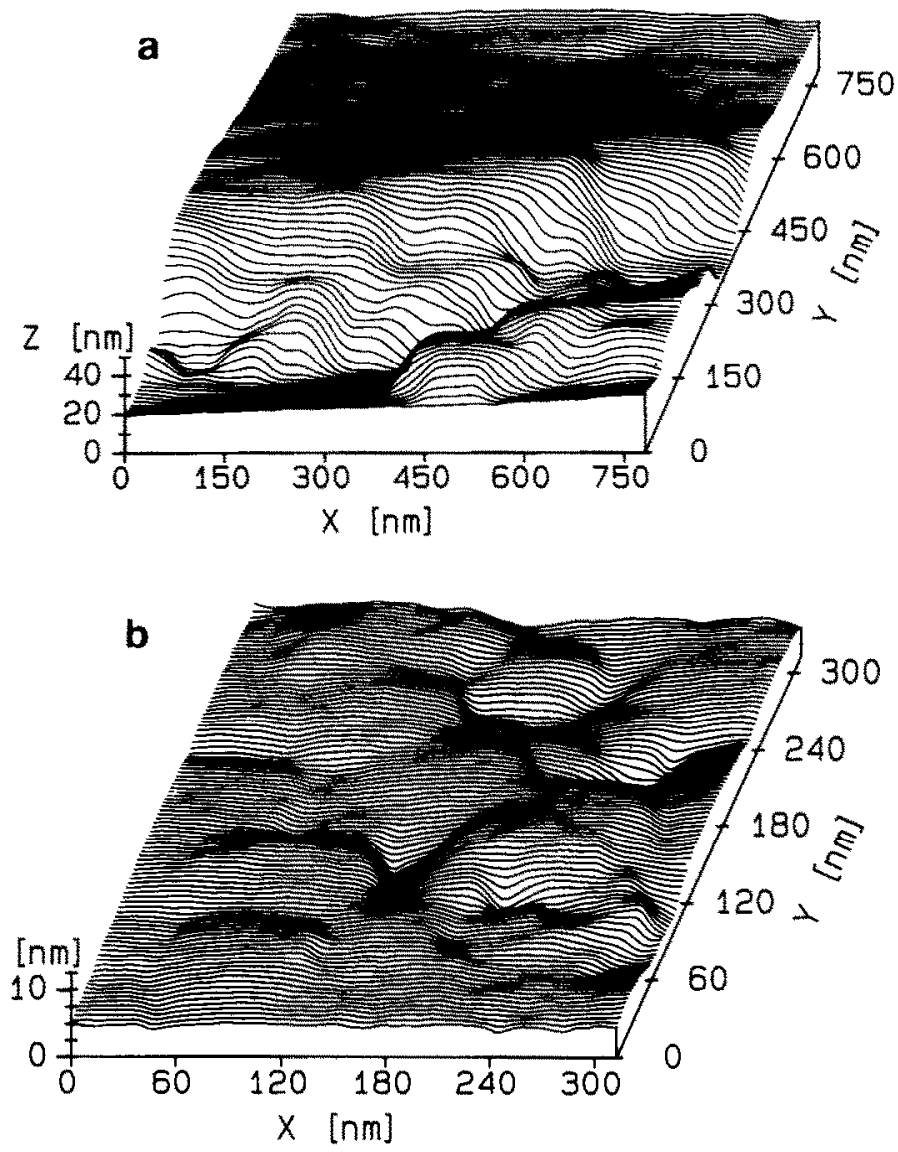

Fig. 9. Mixed surface: Au(80 nm) films on mica: (a) The furrowing of the mica substrates during the cleavage process exemplified by a large area scan $(0.75 \times 0.75 \mu \mathrm{m})$ of the $80 \mathrm{~nm}$ thick gold overlayer. (b) An $0.3 \times 0.3 \mu \mathrm{m}$ area scan with an example of mixed topography. Flat regions located on scaly substrate features (due to the exfoliation of mica) are intermixed with hillock regions.

by grooves and depressions (lateral extension: $100 \mathrm{~nm}$, depth: 5 to $10 \mathrm{~nm}$ ) were observed.

The examples of STM images given in figs. $9 \mathrm{a}$ and $9 \mathrm{~b}$ for our samples on air cleaved natural mica (pegmatitic muscovite - Madagascar) were selected from about 80 surface pictures performed on different surface areas.

Some critical discussion seems to be necessary for the interpretation of the topographical maps of fig. 9. Firstly, the cleavage of mica in air seems to be unable to produce smooth and homogeneous substrates with large lateral extensions. The cleavage process itself is accompanied by a strong electrical 
charge-up [32] and therefore, due to the resulting electrostatic forces, a strong "furrowing" of the cleaved surfaces occurs. A typical example is given in the large area scan $(0.75 \times 0.75 \mu \mathrm{m})$ of fig. 9a. The "Grand-Canyon" whose sides consist of mountains and plateaus of various heights and shapes is a clear example for this process. On the high plateau, however, a rather flat landscape can be observed. The identification of topographical details on this area is unfortunately restricted to $5 \mathrm{~nm}$. A large number of scans have been performed on such flat areas on various sites of the surface. After the subsequent analysis of these scans we are convinced that the film surfaces on mica substrates consist of regions with varying topographical character (mixed surfaces). We cannot specify a typical topographical map, characteristic for these gold surfaces. In the range of some hundred nanometer scan areas not only very smooth areas (level differences lower than $1 \mathrm{~nm}$ ) but also typical polycrystalline surfaces (hillocks in the height range of some $\mathrm{nm}$ ) or in many cases a mixture of both has been observed. Oftenly we observed large (scaly) areas of about 3 to $20 \mathrm{~nm}$ in height due to the exfoliation of mica during the cleaving process. Fig. $9 \mathrm{~b}$ shows a selected example of such a mixed surface.

Here very smooth areas ( $5 \AA$ range) partly located on the scaly areas created by the exfoliation of mica together with flat hillocks of about 60 to 100 $\mathrm{nm}$ lateral extension at 2 to $5 \mathrm{~nm}$ in height can be observed.

The imperfect cleavage of mica in air gives rise to regions with different defect characteristics and densities [32]; therefore different incipient growth mechanisms may coexist on the same substrate surface.

Additionally, it should be noted that the temperature of our substrates $(500 \mathrm{~K})$ was slightly less than the minimal value $(570 \mathrm{~K})$ necessary for producing highly oriented films $[11,31]$. These two deficiencies can be the reason for the observed inhomogeneous surface topography of our gold films on mica. Thus smooth areas and hillock regions (as for example in fig. 9b) can coexist.

A direct comparison of our results with the published work of Chidsey and coworkers [16] seems to be rather difficult due to some differences in the preparation of the films. Although the substrate temperature of $500 \mathrm{~K}$ and the evaporation rate of $0.1 \mathrm{~nm} / \mathrm{s}$ are comparable for both experiments, no information concerning the cleaning of the mica substrates in vacuum prior to the evaporation was given in ref. [16]. Relating to the work of Poppa and Elliot [32] this seems to be a crucial point for the quality of air-cleaved mica substrates. Additionally, the evaporation pressures are different in the two experiments: $10^{-6}$ to $10^{-7}$ mbar in the experiment described in ref. [16], $10^{-8}$ mbar in our case.

A correlation of STM images with structure investigations by TEM and $\mathrm{X}$-ray diffraction given in ref. [16], however, demonstrated clearly that by a proper combination of deposition parameters and substrate quality, epitaxial growth of gold on mica occurs even at the relatively low substrate temperature of $500 \mathrm{~K}$. For our films, however, rather the growth mechanism described by 
Reichelt and Lutz [32] seems to be responsible for the observed surface topography (mixed surface).

Finally, the result given in fig. $9 \mathrm{~b}$ is very instructive: Information resulting from a relatively small area scan is not always characteristic for the whole sample.

\section{Conclusion}

In this work the STM was employed for a systematic study of substrate-dependent surface topography of UHV-evaporated gold films.

To our knowledge, this is the first attempt for a comparative study on this still unsatisfactorily solved problem of thin film investigation. Therefore, we discussed the specific literature directly together with the presentation of our own particular results.

The selection of the material and evaporation conditions is decisive for a comparative study. Gold is an inert metal and therefore very appropriate for STM-imaging in air. Since the films were evaporated simultaneously on different substrates mounted on the same holder, identical evaporation parameters for the different substrates were guaranteed.

Very different topographies in a wide dynamical range concerning the level difference and lateral extensions of topographical features have been established on the surface of gold films of the same thickness $(80 \mathrm{~nm})$.

The surface roughness is induced primarily by the incipient growth mechanism of the films. The examples discussed in this work furnished clear evidence for this matter of fact.

Corning glass and silicon substrates induce rough polycrystalline surfaces with hillocks of about 5 to $20 \mathrm{~nm}$ in height and 40 to $100 \mathrm{~nm}$ lateral extension. If the coagulation was inhibited by chromium nucleation centers the gold surface on glass became quite flat (the roughness was smaller than $4 \mathrm{~nm}$ at large lateral extensions). These surface roughnesses for films on glass agree with earlier results evaluated from the thickness-dependent resistivity.

$\mathrm{NaCl}$ and graphite, known from the literature as promoters of oriented growth, induced very smooth surfaces. Due to the extraordinary surface smoothness of these gold films, features due to the cleavage steps of the underlying single crystalline substrates can be well distinguished from the topography of the films themselves. For gold on graphite we successfully resolved the hexagonal (111) atomic corrugations on a preselected $20 \times 20 \AA$ area of (111) textured films.

The good agreement of the results given in this paper with previous investigations of growth experiments should be viewed as very encouraging. The STM, therefore, gives a real chance to solve the main part of topographical questions in thin film investigations. Finally, the proof of the 
topography on various sites of the sample is absolutely necessary. The example of gold surfaces on mica is very instructive in that regard.

The STM in air can be developed to a powerful routine method for the investigation of coating surfaces. More systematical experiments using other materials are necessary. The recent successful attempts in the imaging of the silicon surface in air [12] shows that a real chance for the assertion of this practice exists.

\section{References}

[1] L. Reimer, Elektronenmikroskopische Untersuchungs- und Präparationsmethoden (Springer, Berlin, 1967).

[2] R.Suhrmann, R. Gerdes and G. Wedler, Z. Naturforsch. 18a (1963) 1211.

[3] Y. Namba, Japan. J. Appl. Phys. 9 (1970) 1326.

[4] J. Vancea, H. Hoffman and K, Kastner, Thin Solid Films 121 (1984) 201.

[5] H. Hoffmann and J. Vancea, Thin Solid Films 85 (1981) 147.

[6] H. Hoffmann, J. Vancea and U. Jacob, Thin Solid Films 129 (1985) 181.

[7] G. Binnig, II. Rohrer, Ch. Gerber and E. Weibel, Phys. Rev. Letters 49 (1982) 57.

[8] B. Marchon, S. Ferrer, D.S. Kaufman, M. Salmeron and W. Siekhaus, Thin Solid Films 154 (1987) 65 .

[9] R.C. Jaklevic, L. Elie, Weidian Shen and J.T. Chen, J. Vacuum Sci. Technol. A 6 (1988) 448.

[10] R. Laiho, T. Levola and H. Snellman, Surface Sci. 181 (1987) 370.

[11] V.M. Hallmark, S. Chiang, J.F. Rabolt, J.D. Swalen and R.J. Wilson, Phys. Rev. Letters 59 (1987) 2879.

[12] K.H. Besocke, M. Teske and J. Frohn, J. Vacuum Sci. Technol. A 6 (1988) 408.

[13] G. Binnig and D.P.E. Smith, Rev. Sci. Instr. 57 (1986) 1688.

[14] Ch. Gerber, G. Binnig, H. Fuchs, O. Marti and H. Rohrer, Rev. Sci. Instr. 57 (1986) 221.

[15] Delivered by Quartz \& Silice, F-77140 St. Pierre les Nemours.

[16] C.E.D. Chidsey, D.N. Loyacono, T. Sleator and S. Nakahara, Surface Sci. 200 (1988) 45.

[17] K.L. Chopra, Thin Film Phenomena (McGraw-Hill, New York, 1969).

[18] T. Andersson and C.G. Granqvist, J. Appl. Phys. 48 (1977) 1673.

[19] S. Norrman, T. Andersson, G. Petö and S. Somogyi, Thin Solıd Films 77 (1981) 359.

[20] M.P. Cox and P.R. Griffin, J. Vacuum Sci. Technol. A 6 (1988) 376.

[21] K.H. Besocke, Surface Sci. 181 (1987) 145.

[22] F. Besenbacher, E. Laesgaard, K. Mortensen, U. Nielsen and I. Stensgaard, Rev. Sci. Instr. 59 (1988) 1035 .

[23] G. Mathieu, R. Contini, J.M. Layet, P. Mathiez and S. Giorgio, J. Vacuum Sci. Technol. A 6 (1988) 294.

[24] G. Reiners, Thin Solid Films 143 (1986) 311.

[25] M. Lauxner, C.L. Bauer and R. Scholz, Thin Solid Fllms 50 (1987) 323.

[26] K.L. Chopra, Thin Film Phenomena (McGraw Hill, New York, 1969) p. 224.

[27] D.W. Pashley, Advan. Phys. 14 (1965) 327.

[28] J.W. Matthews, Epitaxial Growth, Part A and B (Academic Press, New York, 1975).

[29] T.P. Darby and C.M. Wayman, Phys. Status Solidi (a) 1 (1970) 729.

[30] E. Ganz, K. Sattler and J. Clarke, J. Vacuum Sci. Technol. A 6 (1988) 419.

[31] K. Reichelt and H.O. Lutz, J. Crystal Growth 10 (1971) 103.

[32] H. Poppa and A.G. Elliot, Surface Sci. 24 (1971) 149.

[33] K.L. Chopra, Thin Film Phenomena (McGraw Hill, New York, 1969) p. 182. 